\title{
RICCI CURVATURE AND A CRITERION FOR SIMPLE-CONNECTIVITY ON THE SPHERE
}

\author{
MARTIN CHUAQUI
}

(Communicated by Christopher Croke)

\begin{abstract}
From the recent work of Osgood and Stowe on the Schwarzian derivative for conformal maps between Riemannian manifolds we derive a sharp sufficient condition for a domain on the sphere to be simply-connected. We show further that a less restrictive form of the condition yields a uniform lower bound for the length of closed geodesics.
\end{abstract}

\section{INTRODUCTION}

Osgood and Stowe have recently defined a notion of Schwarzian derivative for conformal mappings of Riemannian manifolds which generalizes the classical operator for analytic functions in the plane [O-S1]. As in complex analysis, where the Schwarzian derivative has been central as a means of characterizing conditions for global univalence, these authors establish in [O-S2] an injectivity criterion for conformal local diffeomorphisms $\psi$ of a Riemannian $n$-manifold $(M, g)$ to the standard sphere $S^{n}$. The univalence of $\psi$ follows from a bound on the norm of the Schwarzian derivative by geometric quantities of $M$ (Theorem 1.1). This result allows a unified approach to a vast class of injectivity theorems in the plane, as different criteria can be derived from it on a given domain just by changing the metric $g$ conformally. Indeed, in [O-S2] the authors obtain as corollaries, with $M$ the unit disc in the plane and $g$ alternately the euclidean and hyperbolic metric, two classical conditions of Nehari. Most of the known criteria, including a recent injectivity result of Epstein [Ep], and some new conditions on the unit disc and simply-connected domains are derived in [Ch1] from Theorem 1.1.

We shall show in this paper that a local diffeomorphism $\psi$ as before satisfying a particular form of the criterion in [O-S2] forces the manifold $M$ to be simply-connected (Theorem 2.1). Our main result, a sharp criterion for simpleconnectivity for domains in $S^{n}$, will appear as a reformulation of Theorem 2.1 when using conformal invariance. This allows one to translate the existence of $\psi$ to that of a conformal metric on a domain $\Omega \subset S^{n}$ with the property that

Received by the editors October 9, 1991 and, in revised form, January 8, 1993.

1991 Mathematics Subject Classification. Primary 53A30; Secondary 34C10.

Key words and phrases. Schwarzian derivative, univalence, conformal metric, simple-connectivity, closed geodesic, trace-free Ricci, scalar curvature. 
the norm of the trace-free Ricci tensor is bounded above by a dimensional constant multiple $c_{n}$ of the scalar curvature. The effect of changing the constant $c_{n}$ to $c$ is, in our opinion, quite remarkable. With $c<c_{n}$ one can construct a reflection $\Lambda: S^{n} \rightarrow S^{n}$ which maps $\Omega$ to $S^{n}-\bar{\Omega}$ and which fixes pointwise $\partial \Omega$ [Ch2]. The mapping $\Lambda$ is quasiconformal in the sense of Ahlfors, i.e., the ratio of the largest and smallest eigenvalue of the symmetrized differential $(D \Lambda)(D \Lambda)^{t}$ is uniformly bounded. Here we show that for $c>c_{n}$ the criterion yields a uniform lower bound for the length of closed geodesics in $\Omega$.

\section{Preliminaries}

We shall present in this section enough of the work in [O-S1] so that we can state the injectivity criterion in [O-S2]. We will omit proofs and refer the reader to the sources for more details.

Let $M$ be an $n$-dimensional Riemannian manifold with metric $g$. When $M=R^{n}$, we will denote by $g_{0}$ the euclidean metric and $g_{1}$ will stand for the standard metric on the sphere $S^{n}$.

Given a conformal metric $\hat{g}=e^{2 \varphi} g$ on $M$, Osgood and Stowe define the Schwarzian tensor of $\hat{g}$ with respect to $g$ as the symmetric, trace-free $(0,2)$ tensor

$$
B_{g}(\varphi)=\operatorname{Hess}(\varphi)-d \varphi \otimes d \varphi-\frac{1}{n}\left(\Delta \varphi-|\operatorname{grad} \varphi|^{2}\right) g
$$

where the metric dependent quantities on the right-hand side are computed with respect to the metric $g$. We mention here that the tensor $B_{g}(\varphi)$ appears as the term by which the trace-free part of the Ricci tensor changes under the conformal change of metric $g$ to $e^{2 \varphi} g$. When $\psi$ is a conformal local diffeomorphism of $(M, g)$ to another Riemannian manifold $\left(N, g^{\prime}\right)$, then $\psi^{*}\left(g^{\prime}\right)=e^{2 \varphi} g$ with $\varphi=\log |D \psi|$. The Schwarzian derivative of $\psi$ is defined by

$$
S_{g}(\psi)=B_{g}(\varphi) \text {. }
$$

For an analytic map $\psi$ in the plane, with $g=g^{\prime}=g_{0}, \varphi=\log \left|\psi^{\prime}\right|$. Computing in standard coordinates one gets

$$
S_{g}(\psi)=\left(\begin{array}{cc}
\operatorname{Re}\{\psi, z\} & -\operatorname{Im}\{\psi, z\} \\
-\operatorname{Im}\{\psi, z\} & -\operatorname{Re}\{\psi, z\}
\end{array}\right),
$$

where $\{\psi, z\}=\left(\frac{\psi^{\prime \prime}}{\psi^{\prime}}\right)^{\prime}-\frac{1}{2}\left(\frac{\psi^{\prime \prime}}{\psi^{\prime}}\right)^{2}$ is the classical Schwarzian derivative.

On $M$, the conformal metric $\hat{g}=e^{2 \varphi} g$ is called Möbius with respect to $g$ if $B_{g}(\varphi)=0$, and so a conformal local diffeomorphism $\psi$ is said to be Möbius if $S_{g}(\psi)=0$. If $\varphi$ and $\sigma$ are smooth functions on $M$, then there is an important identity:

$$
B_{g}(\varphi+\sigma)=B_{g}(\varphi)+B_{\hat{g}}(\sigma)
$$

where $\hat{g}=e^{2 \varphi} g$. In a chain of conformal local diffeomorphisms $\psi_{1}:(M, g) \rightarrow$ $\left.N_{1}, g^{\prime}\right)$ and $\psi_{2}:\left(N_{1}, g^{\prime}\right) \rightarrow\left(N_{2}, g^{\prime \prime}\right)$, equation (1.1) can be formulated as

$$
S_{g}\left(\psi_{2} \circ \psi_{1}\right)=S_{g}\left(\psi_{1}\right)+\psi_{1}^{*}\left(S_{g^{\prime}}\left(\psi_{2}\right)\right) \text {. }
$$

This recovers the classical formula of the Schwarzian derivative of a composition of analytic maps in the plane. 
By $\left\|B_{g}(\varphi)\right\|$ we mean the norm of the Schwarzian tensor $B_{g}(\varphi)$ with respect to $g$, as a bilinear form on each tangent space, that is,

$$
\left\|B_{g}(\varphi)\right\|=\max \left\{\left|B_{g}(\varphi)(X, Y)\right|:|X|=|Y|=1\right\} .
$$

In some cases, we will need to consider the norm of $B_{g}(\varphi)$ in a metric $\hat{g}=e^{2 \sigma} g$ conformal to $g$. Then

$$
\left\|B_{g}(\varphi)\right\|_{\hat{g}}=e^{-2 \sigma}\left\|B_{g}(\varphi)\right\| .
$$

With this, we present the theorem in [O-S2].

Theorem 1.1. Let $(M, g)$ be a Riemannian manifold of dimension $n \geq 2$ and $\psi:(M, g) \rightarrow\left(S^{n}, g_{1}\right)$ a conformal local diffeomorphism. Suppose that the scalar curvature of $M$ is bounded above by $n(n-1) K$ for some $K \in R$, and that any two points in $M$ can be joined by a geodesic of length $<\delta$ for some $0<\delta \leq \infty$. If

$$
\left\|S_{g}(\psi)\right\| \leq \frac{2 \pi^{2}}{\delta^{2}}-\frac{1}{2} K
$$

then $\psi$ is injective.

With $M$ the unit disc in the plane and $g$ alternately the euclidean and hyperbolic metric, Osgood and Stowe derive from this theorem the classical criteria of Nehari; namely, that

$$
|\{\psi, z\}| \leq \frac{\pi^{2}}{2} \quad \text { or } \quad|\{\psi, z\}| \leq \frac{1}{\left(1-|z|^{2}\right)^{2}}, \quad \text { all }|z|<1,
$$

imply that $\psi$ is injective.

We point out that in Theorem 1.1 , the target $\left(S^{n}, g_{1}\right)$ can be replaced by the standard hyperbolic space $H^{n}$ or $\left(R^{n}, g_{0}\right)$. This follows from the transformation law (1.1) and the fact that both $g_{1}$ and the hyperbolic metric are Möbius with respect to the euclidean metric. Finally, let $\operatorname{scal}(g)$ be the scalar curvature of $g$. It is easy to see that the proof given by Osgood and Stowe works equally well when assuming that at each point in $M$ the norm of the Schwarzian derivative of $\psi$ is bounded above by

$$
\frac{2 \pi^{2}}{\delta^{2}}-\frac{\operatorname{scal}(g)}{2 n(n-1)} \text {. }
$$

\section{A CRITERION FOR SIMPLE-CONNECTIVITY}

To begin with, we note the following consequence of Theorem 1.1.

Theorem 2.1. Let $(M, g)$ be a complete Riemannian manifold of dimension $n \geq 2$ and $\psi:(M, g) \rightarrow\left(S^{n}, g_{1}\right)$ a conformal local diffeomorphism. If

$$
\left\|S_{g}(\psi)\right\| \leq-\frac{\operatorname{scal}(g)}{2 n(n-1)}
$$

then $M$ is simply-connected.

Even though one can give an independent proof of this theorem, it will follow from Theorem 2.2. Let us consider the image $\Omega=\psi(M)$. Under the 
hypotheses of Theorem 2.1, $\psi$ is injective and we let $g_{2}=e^{2 \rho} g_{1}=\phi^{*}(g)$, where $\phi=\psi^{-1}$. The addition formula (1.2) implies

$$
S_{g}(\psi)=-\psi^{*}\left(S_{g_{1}}(\phi)\right)=-\psi^{*}\left(B_{g_{1}}(\rho)\right)
$$

and therefore

$$
\left\|S_{g}(\psi)\right\|=\left\|B_{g_{1}}(\rho)\right\|_{g_{2}} .
$$

Our main result is

Theorem 2.2. Let $\Omega \subset S^{n}$ be a domain with a complete metric $g_{2}=e^{2 \rho} g_{1}$. If

$$
\left\|B_{g_{1}}(\rho)\right\|_{g_{2}} \leq-\frac{\operatorname{scal}\left(g_{2}\right)}{2 n(n-1)}
$$

then $\Omega$ is simply-connected.

Proof. Let $\tilde{\Omega}$ be the universal cover of $\Omega$ with covering map $\pi$ and metric $\tilde{g}=\pi^{*}\left(g_{2}\right)$. We consider $\pi$ as a conformal map from $(\tilde{\Omega}, \tilde{g})$ into $\left(S^{n}, g_{1}\right)$. We shall show that

$$
\left\|S_{\tilde{g}}(\pi)\right\|=\left\|B_{g_{1}}(\rho)\right\|_{g_{2}},
$$

which by Theorem 1.1 implies the univalence of $\pi$ and, consequently, the theorem.

We have

$$
\pi^{*}\left(g_{1}\right)=\pi^{*}\left(e^{-2 \rho} g_{2}\right)=e^{-2(\rho \circ \pi)} \tilde{g}
$$

hence

$$
S_{\tilde{g}}(\pi)=B_{\tilde{g}}(-\rho \circ \pi)=\pi^{*}\left(B_{g_{2}}(-\rho)\right)=-\pi^{*}\left(B_{g_{1}}(\rho)\right) .
$$

This proves (2.1).

Remarks. (1) Theorem 2.2 can be stated as well for domains in $R^{n}$ with $g_{0}$ as the background metric. The hypotheses of the theorem implicitly require that $\operatorname{scal}\left(g_{2}\right) \leq 0$. Moreover one can show that $g_{2}$ has nonpositive curvature. Indeed, since $g_{2}$ is conformally flat, its Weyl tensor vanishes, and now a classical decomposition of the Riemann curvature tensor allows us to compute sectional curvatures solely in terms of $\operatorname{scal}\left(g_{2}\right)$ and the trace-free part of the Ricci tensor. If $X, Y$ are orthonormal tangent vectors in the metric $g_{2}$, then the sectional curvature $K(X, Y)$ of $g_{2}$ is given by

$$
K(X, Y)=\frac{\mathrm{scal}\left(g_{2}\right)}{n(n-1)}+B_{g_{1}}(\rho)(X, X)+B_{g_{1}}(\rho)(Y, Y) .
$$

Therefore $K(X, Y) \leq 0$ and so $\Omega$ as in the theorem is actually diffeomorphic to $R^{n}$.

(2) The condition that $g_{2}$ be complete can be relaxed; what one really needs is that any two points in $\widetilde{\Omega}$ can be joined by some geodesic in the metric $\tilde{g}$. Also, the theorem can be stated slightly more generally: without the assumption of completeness, if

$$
\left\|B_{g_{1}}(\rho)\right\|_{g_{2}} \leq \frac{2 \pi^{2}}{\delta^{2}}-\frac{\operatorname{scal}(g)}{2 n(n-1)}
$$

then there are no closed (not even nonsmoothly closing) geodesics in the metric $g_{2}$ of length $<\delta$. 
(3) Theorem 2.2 is sharp. For $n=2$ this can be verified by taking in the plane the ring $R_{1}<|z|<R_{2}$ with its Poincaré metric $g=e^{2 \varphi} g_{0}$. This metric satisfies the inequality

$$
\left\|B_{g_{0}}(\varphi)\right\|_{g} \leq-(1+\varepsilon) \frac{\operatorname{scal}(g)}{4}
$$

where $\varepsilon\left(\log \left(R_{2} / R_{1}\right) / \pi\right)^{2}$ can be made arbitrarily small.

In higher dimensions we consider a similar example: a hyperbolic solid torus. Let $n \geq 2$ and let $\Omega$ be the domain in $R^{n+1}$ given by

$$
\left\{\left(x_{1} \cos \theta, x_{1} \sin \theta, x_{2}, \ldots, x_{n}\right):\left(x_{1}-a\right)^{2}+x_{2}^{2}+\cdots+x_{n}^{2}<1\right\},
$$

where $a>1$. To simplify notation, we write $r^{2}=\left(x_{1}-a\right)^{2}+x_{2}^{2}+\cdots+x_{n}^{2}$. Let $g=e^{2 \varphi} g_{0}$ with $\varphi=-\log \left(1-r^{2}\right)$. This metric is complete and we will show that given $\varepsilon>0$, the inequality

$$
\left\|B_{g_{0}}(\varphi)\right\|_{g} \leq-(1+\varepsilon) \frac{\mathrm{scal}(g)}{2 n(n+1)}
$$

will hold throughout $\Omega$ provided the constant $a$ is sufficiently large. With respect to the coordinates $x_{1}, \ldots, x_{n}, \theta$ the tensor $B_{g_{0}}(\varphi)$ is a diagonal matrix with eigenvalues $\lambda=\lambda_{1}=\cdots=\lambda_{n}$ and $\lambda_{n+1}$ given by

$$
\lambda=\frac{2 a}{(n+1)\left(1-r^{2}\right) x_{1}}
$$

and

$$
\lambda_{n+1}=\frac{-2 n a x_{1}}{(n+1)\left(1-r^{2}\right)} .
$$

A standard formula gives

$$
\begin{aligned}
-e^{2 \varphi} \frac{\operatorname{scal}(g)}{n(n+1)} & =\frac{2}{n+1} \Delta \varphi+\frac{n-1}{n+1}|\operatorname{grad} \varphi|^{2} \\
& =\frac{4}{\left(1-r^{2}\right)^{2}}-\frac{4 a}{(n+1)\left(1-r^{2}\right) x_{1}} .
\end{aligned}
$$

The vector fields $\frac{\partial}{\partial x_{i}}$ have euclidean length 1 while $\frac{\partial}{\partial \theta}$ has length $x_{1}$. It follows that $\left\|B_{g_{0}}(\varphi)\right\|_{g}=e^{-2 \varphi} x_{1}^{-2} \lambda_{n+1}$. If $c=1+\varepsilon$ then the desired inequality is

$$
\frac{2 n a}{(n+1)\left(1-r^{2}\right) x_{1}} \leq(1+\varepsilon)\left\{\frac{2}{\left(1-r^{2}\right)^{2}}-\frac{2 a}{(n+1)\left(1-r^{2}\right) x_{1}}\right\}
$$

which simplifies to

$$
\frac{(n+1+\varepsilon) a}{(n+1) x_{1}} \leq \frac{1+\varepsilon}{1-r^{2}} .
$$

Since $a-1<x_{1}$ the last inequality will hold if $a \geq \frac{(n+1)(1+\varepsilon)}{n \varepsilon}$.

\section{SHORT GEODESICS}

The proof of Theorem 1.1 relies on translating the given inequality on $\psi$ to a differential inequality along geodesics of a suitably chosen test function $w$. To be more precise, let $\gamma$ be a geodesic joining two given points $x, y \in M$. 
The function $w$ is nonnegative and constructed so that it vanishes at $p$ if and only if $\psi(x)=\psi(p)$ [O-S2]. Along $\gamma$

$$
w^{\prime \prime} \geq-w\left(\left\|S_{g}(\psi)\right\|+\frac{\operatorname{scal}(g)}{2 n(n-1)}\right)+\frac{\left(w^{\prime}\right)^{2}}{2 w}
$$

whenever $w>0$. The estimate on $\left\|S_{g}(\psi)\right\|$ as in Theorem 2.2 implies that $\left(w^{1 / 2}\right)^{\prime \prime} \geq 0$ and therefore $\psi(x) \neq \psi(y)$.

We assume now that $g$ is complete and consider the case when $\psi$ satisfies the estimate

$$
\left\|S_{g}(\psi)\right\| \leq-c \frac{\operatorname{scal}(g)}{2 n(n-1)}
$$

for some $c>1$. Suppose also that $\frac{\operatorname{scal}(g)}{n(n-1)} \geq-s>-\infty$. Then

$$
\left(w^{1 / 2}\right)^{\prime \prime} \geq-\frac{(c-1) s}{4} w^{1 / 2} .
$$

A standard Sturm comparison theorem guarantees that $w$ cannot vanish again before time

$$
d=\frac{2 \pi}{\sqrt{s(c-1)}} .
$$

In other words, if $\psi(x)=\psi(y)$ for $x \neq y$, then the distance between these two points is at least equal to $d$. We reformulate this as:

Theorem 3.1. Let $\Omega \subset S^{n}$ be a domain with a complete metric $g_{2}=e^{2 \varphi} g_{1}$. Assume that

$$
-\infty<-s \leq \frac{\operatorname{scal}(g)}{n(n-1)} \leq 0
$$

If for some $c>1$

$$
\left\|B_{g_{1}}(\varphi)\right\|_{g_{2}} \leq-c \frac{\operatorname{scal}(g)}{2 n(n-1)}
$$

then any closed geodesic in $\Omega$ has length at least $d$.

The ring domain $R_{1}<|z|<R_{2}$ with its hyperbolic metric shows that (3.1) is sharp.

\section{ACKNOWLEDGMENTS}

I would like to thank the referee for pointing out some mistakes in the original computations in the main example, as well as for other thoughtful comments.

\section{REFERENCES}

[Ch1] M. Chuaqui, A unified approach to univalence criteria in the disc and simply-connected domains, Proc. Amer. Math. Soc. (to appear).

[Ch2] _ The Schwarzian derivative and quasiconformal reflections on $S^{n}$, Ann. Acad. Sci. Fenn. Ser. A I Math. 17 (1992), 315-326.

[Ne] Z. Nehari, The Schwarzian derivative and schlicht functions, Bull. Amer. Math. Soc. 55 (1949), 545-551.

[Ep] C. Epstein, The hyperbolic Gauss map and quasiconformal reflections, J. Reine Angew. Math. 372 (1986), 96-135. 
[O-S1] B. Osgood and D. Stowe, The Schwarzian derivative and conformal mapping of Riemannian manifolds, Duke Math. J. 67 (1992), 57-99.

[O-S2] _ A generalization of Nehari's univalence criterion, Comment. Math. Helv. 65 (1990), 234-242.

Department of Mathematics, University of Pennsylvania, Philadelphia, PennsylvaNIA 19104

Current address: Faculty of Mathematics, P. Universidad Catolica de Chile, Casilla 306, Santiago 22, Chile

E-mail address: mchuaqui @mat.puc.cl 